\title{
Vom türkischen Kriegsschauplatz.
} II.

Der Dienst der Truppen an der Front wurde von den Bataillonsärten ulit dell Sanitätssoldaten versehen. Es entspricht nicht deu Tat. sachen, daß ein Aerztemangel vorlag, in Gegenteil waren meist mehr als genug da, was ja bei der Zusammenpressing der sich zurückziehenden Truppenkörper erklärlich ist. Ebenso entspricht es nicht den Tatsachen, daß es im allgeneinen an Sanitätsuaterial, vor allem an Verband. zeug. gefehlt habe. Das war nud ist reichlich vorhanden, allen Zeitungsnachrichten zum Trotz. Das schließt aber nicht aus, daß Personal und Material, sei es aus Mangel an Unisicht, sei es infolge schlechter Beschaffungsmöglichkeiten, einmal nicht zur Stelle war. Um die Zahl der aktiveı Militärärzte zu vermehren, wurden die außer Dienst gestellten wieder einberufen, Zivilärzte engagiert (die allgemeine Wehrpflicht war bei den Aerzten noch nicht durchgeführt, da ein entsprechender Gesetzentwurf vonı Parlament noch nicht erledigt war), und auf die Medizinalpraktikanten des Zivil- und Militärstandes sowie auf die letzte Klasse der Medizinschüler zurückgegriffen, während die Medizinschüler der beiden vorhergehenden Klassen als Assistenten und Gehilfen auf die Krankenhäuse verteilt wurden.

An geschultem Pflegepersonal fehlte es sehr. Wohl wurclen zu Beginn des Krieges rasch Kurse für türkische und ausländische Dauneu und Herren eingericlitet, aber damit war der Bedarf doch nicht zu decken, und den wenigen Schwestern in den verschiedenen Krankenhäusern fiel eine ungeheure Arbeitslast zu. Auch dem Roten Halbmond, der während des ganzen Krieges nit Eifer und Aufopferıng gearbeitet hat, fehlte es. all einheinischenı Personal. Höchst willkommen waren darım die verschiedenen Expeditionen des Roten Kreuzes, wenngleich allch sie ja bei weitem den Bedarf besonders an sprachenkundigem Persoulal nicht decken konuten. Eine sehr wertvolle Hilfe fauden wir an den Damen der verschiedenen Kolonien, die sprachkundig, praktisch und fleißig ihie freiwillig übernommenen Aufgaben lösten; ihnen dankt das Krankenwesen viel!

Ich nöchte hier der Roten Kreuze kurz gedenken, ihnen dauken für die anfopferungsvolle Hilfe, die sie dem Lande geleistet haben. Ich habe nich nach Kräften bemüht, für sie zll sorgen, ohne Ulterschied der Nation. Wenn die deutsche Expedition, wie Dr. Schleip in der Mün. chener medizinischen Wochenschrift sagt, am vorteilhaftesten unterge bracht war, so lag das daran, daß sie zuerst kam, woch zu einer Zeit wo Bedarf vorhanden war. Die englische Rote Kreuz-Expedition sie war übrigens privater Natur - war besonders gnt für das Feld ausgeriistet. Die ineisten Missionen kamen so spät -- oder der Krieg war so früh zu Ende, daß wenig Verwundete für sie übrig blieben. Auf den kleinen Raum in Konstantinopel waren alle Missionen zusammengedrängt die meisten wollten nur ,Verwuudete" haben, nur ungern innere Krankheiten, die eillen warell nur für den Hospital-Operationsbetrieb, die andern nur für das Feld ausgerüstet. Branchbar für alle Zwecke wa nur eine englische, eine ägyptische und eine rumänische Mission aus gerüstet, die Zelte oder Baracken und alles nötige Zubehör mitfïhrte Es sind auf beiden Seitel Fehler begangen worden. Um wirklich Zweck entsprechendes zu lcisten, und das will mall doch, muß Bedarf und Darbietung sich eutsprechen, müssen dortige Wünsche und hiesige Bediirfnisse in Einklang stehen oder doch wenigstens bekannt sein. Aut tïrkischer Seite fehlte anfangs die nötige Erfahrung in der Organisation der freiwilligen Krankenpflege. Rote Kreuz- und Privatpersonen okku pierten anderseits Gebiete, zu denen sie nicht berufen waren. Einzel. personen und Gruppen tauchten auf und wirktell, ohne daß die offizielle Behörde davon benachrichtigt, geschweige denn darım gebeten war. Ich habe nit allen Kräften versucht, da Ordnung $\mathrm{zu}$ schaffen, und habe betont, daß im Kriege die Verwendung von Hilfsexpeditionen nur mit Einverständnis oder nach Bestimmung der offiziellen Behörde des Kricgs ministeriums resp. des Chefs des Medizinalwesens erfolgen dürfe; be Leibe nicht, un den humanitären Bestrebungen entgegen zu treten, sondern nur um sie in die rechtel Wege zu leiten und aln richtigen Orle, unter Vernieidung unnützer Kraftvergeudung und nationaler oder persönlicher Differenzen einzusetzen.

Mir ist das nicht ganz gelungen, und ich habe zuın Dank z. B. von ausländischen hochgestellten Personen, die es anders hätten wissen 
sollen, gehört, ,,ich suche die humanitär'en Bestrebıngen der freiwilligenı Krankenpflege zu unterdrückeı, da das Militär'sanitätswesen allein be rufen sei, sie zu üben", während gerade ich von Anfang an und init allen Kräften die fremden Roten Krelız-Expeditionen hreanzuziehen und den Roten Halbmond zu unterstützen bemüht war. Ein holländischer Berichterstatter des ,Nieuwe Rotterdamsche Courant" ging so weit, uns deutschen Aerzten die schwersten Vorwürfe ins Gesicht zu schleudern ınd ıns wegen Hintertreibıng der humanitären Bestrebungen - coweit sie nicht dentsche wären - gewissermaßen zum Massenmörder zu stempeln! Als der Krieg begann, wurde Dr. Hadji Em in Pascha, ein älterer erfahrener und sehr tätiger Mann. zuın Chef des Feldsanitätsdienstes eriannt, ich ihm als Berater lind zugleich als Berater der Ostarmec beigegeben, mit dem Sitz, ,einstweilen" in Konstantinopel. Oberst $V$ oll bre cht B e y wurde in gleicher Eigenschaft der Westarmee zugewiesen; cr hat sie allerdings nie erreicht, denn bis die Formalitäten seiner aktiven Betciligung erledigt und die Reiseerlanbnis erwirkt war, waren die Wege nach Saloniki verlegt, ihm wie auch anderen, z. B. der zweiten deutschen ınd der rumänischen Hilfsexpedition. So muBte er wohl oder äbel bleiben, nnd wir behielten an ihm einen stets hilfsbereiten Ratgeber und im Krankenhause einen kräftigen Mitarbeiter. Hätten Oberst Voll l, recht und Prof. Richard Bier nicht bereitwilligst sich der Gülhaneanstalt zui Verfügung gestellt, so hätten wir das nicht leisten können, was geleistet wurde; doch davon einmal später — wir gedenken gemeinsam ïber unsere Kriegserfahrungen zu berichten.

Ich möchte hier nicht die Frage der guten und schlechten Behandlıng der deıtschen, im türkischen Dienst stehenden Instruktionsoffiziere behandeln, muß aber doch darauf anfmerksam machen, daß man hierzu lande wie anderwärts auch den gerade nuaßgebenden Stellen nicht allougroße Gesichtspunkte nuterschieben sollte: die tiefsten sozialen und politischen Probleme laufen häufig in rcin pcrsönliche Bcziehıngen aus; was heute oben, ist norgen unten. Oberst Voll brecht Be y, der als Reorganisator des Sanitätswesens berufen war, hat stets freie Bahn gehabt, und ich persönlich - ich rechne mich freilich nicht zu delı Instruktionsoffizieren, da meine eigentliche Tätigkcit mchr auf rein medizinischem Gebiet liegt - kann mich nicht beklagen, daß man mir jemals in der Türkei die nötige Achtung vorenthalten habe. Auf Rosen ist man freilich nicht gebettet; Schwierigkeiten sind täglich in Menge zu überwinden. Dank verlangten wir nie, nur das Recht, unsere Pflicht erfïllen zı können, und so können wir auch nicht von Undank reden. Besonders in diesem Kriege fanden wir stets bereitwilliges Entgegenkommen.

Noch vor der offiziellen Kriegserklärung wurde von mir der Etappendienst bei der Ostarmee eingerichtet. Von Stambul bis Dedeagatsch und darüber hinsaus bis Gümüedschina wurden Erfrischungsstationen, Etappenlazarette, Aufnahmestationen und Depots angelegt. Da die Eisenbahn zum großen Teil parallel zur Aufmarschlinie lief, war der Etappendienst nicht allzu schwer. Aerzte, Sanitätsmaterial, Waggons nach dem Linxweilersehen System - das sich vortrcfflich bewährte, aber leider in zil geringer Anzahl vorhanden war - waren auf die Hauptstationen verteilt, bis wohin der Abtransport von der Front mit Lebensmittel- oder Bauernwagen geschehen mußte. Am Bahnhof in Stambul ist eine Aufnahmestation eingerichtet, die für den Abtransport in die Hospitäler sorgt, nachdein die Erfrischungsstation des Roten Halbmondes alle Ankommenden mit suppe, Tee und Brot versorgt hat.

Leider wurde der Rückzıg zıl rasch vollzogen, sodaß die Etappenstationen eine nach der anderen verschwanden, bis in der Tschataltschalinie Halt gemacht war. So ward der Etappendienst in der Ausdehnung wohl einfacher, in der Ausführung aber schwicrigcr gcworden, wie es ja bei einer im Rückzug begriffenen Armee erklärlich ist. Die von der Front heimkehrenden Züge der eingleisigen Bahn, die bei siegreichem Vorrücken leer geblieben wären, waren jetzt iiberfüllt mit zurückgeworfenem Material und flichenden oder schwachen oder kranken Menschen. Das konnte die eingleisige Bahn, die nicht für Militär'zwecke eingerichtet war, nicht leisten, und so litt das ganze Transport- und Sanitätswesen enorm. Erst in der letzten Zeit ist es möglich geworden, einen Sanitätszug täglich regelmäßig bis Hademköi und Stambul verkehren zu lassen. Dic Strecke von $54 \mathrm{~km}$, die sonst in zwei Stınden $z$ irïckgelegt wird, hat oft $3 \times 24$ Stunden in Anspruch genommen, während welcher Zeit cine regelrechte Versorgung der Heimkehrenden nicht stattfinden konnte. Die'Zahl der Verwundeten, die vom 21. Oktober bis 3. Dezember mit der Bahn in Stambul eintrafen, war rund 17200 , dazı kainen per Schiff etwa 500, sodaß wil rund 18000 wirklich Verwundete rechnen können. Weiter traf später ilı gleichem Zeitraum die doppelle Anzahl ${ }^{1}$ ) Mannschaften mit inneren Krankheiten (Cholera, Dysenterie ctc.), Schwachen, Feigen iiber Stambul und über San Stefano ein. Wenn man nun bedenkt, $\mathrm{da} B$ diese Menschenmasse abermals um das Doppelte vermehrt wurde clurch flïchtende Bauern, dic mit Weib und Kind, mit Sack und Pack in, auf und an den Waggons, selbst den Lazarettwaggons, nach der Hauptstadt eilten, da $\beta$ alle Flüchtlingc, Verwundeten, Cholerakranken und Descrteure in einem und demselben Zuge ankamen, und rasch untergebracht werden wollten, und wenn man vor allem bedenkt, daß diese Menschen

1) Die genauen Zahlen werde ich später mitteilen. nicht in regelmäßigen Abständen, sondern plötzlich wie ein Heuschreckenschwarm einfieleıl, $z$. B. 8000 Verwundete innerhalb drei Tagen, und später dic doppelte Zahl cholcrakranker resp. mit ihnen in Kontakt gewesener śchwächlinge in kaum einer Woche, so wird man sich ein Bild davon ınachen können, wie es damals aussah und welche gewaltige Aufgabe dem Nanitätswesen zufiel. Prof. Wieting Pascha (Konstantinopel). 